\title{
Navier-Stokes simulations in gappy PIV data
}

\author{
Andrea Sciacchitano ${ }^{1, *}$, Richard P. Dwight ${ }^{1}$, Fulvio Scarano ${ }^{1}$ \\ ${ }^{1}$ Faculty of Aerospace Engineering, Delft University of Technology, Delft, The Netherlands \\ *correspondent author: a.sciacchitano@tudelft.nl
}

\begin{abstract}
Velocity measurements conducted with particle image velocimetry (PIV) often exhibit regions where the flow motion cannot be evaluated. The principal reasons for this are the absence of seeding particles or limited optical access for illumination or imaging. Additional causes can be laser light reflections and unwanted out-of-focus effects. As a consequence, the velocity field measured with PIV contains regions where no velocity information is available, i.e. gaps.

This work investigates the suitability of using the unsteady incompressible Navier-Stokes equations to obtain accurate estimates of the local transient velocity field in small gaps; the present approach applies to time-resolved two-dimensional experiments of incompressible convection dominated flows. The numerics are based on a finite volume discretization with partitioned time-stepping to solve the governing equations in the incompressible regime. The measured velocity distribution at the gap boundary is taken as time-varying boundary condition, and an approximate initial condition (I.C.) inside the gap is obtained via low-order spatial interpolation of the velocity at the boundaries. The influence of this I.C. is seen to diminish over time, as information is convected through the gap. Due to the form of the equations, no initial or boundary conditions on the pressure are required. The approach is evaluated by a time-resolved experiment where the true solution is known a-priori. The results are compared with a boundary interpolation approach. Finally, an application of the technique to an experiment with a gap of complex shape is presented.
\end{abstract}

\section{Introduction}

PIV data is often affected by gaps, i.e. regions where the particle image displacement cannot be evaluated and no information on the flow motion is gathered. The main causes of gaps are: 1) an absence of seeding particles due to inhomogeneous tracer dispersion or centrifugal forces; 2) laser light reflections close to objects surface; 3) shadows produced by solid objects in the light path; 4) limited optical access for the imaging system; 5) unwanted out-of-focus effects. The presence of gaps in PIV data is an important issue in large experimental campaigns performed in a limited time-frame and in applications that aim to compute integral quantities. Two examples of the latter are the determination of unsteady aerodynamic loads (Kurtulus et al. 2007) and the computation of acoustic pressure fluctuations in turbulent flows using aeroacoustic analogies (Violato and Scarano, 2011). Despite its relevance, the treatment of gaps has received little to no attention among the PIV community, the problem being regarded as a surrogate of spurious vector detection (e.g. Westerweel and Scarano, 2005) and replacement (Garcia, 2011).

When time permits optimizing the experimental setup, hardware solutions can be employed such as using fluorescent particles (in liquid flows), covering the model surface and test section with a highly absorbing coating (black matt paint), illuminating the model from at least two different angles (to illuminate regions blocked by the model). Software procedures based on image pre-processing have been developed to attenuate or eliminate laser light reflections (Theunissen et al. 2009, Scarano and Sciacchitano, 2011). Furthermore, several data refill procedures have been proposed, based purely on 
spatial interpolation of neighbouring vectors (Gunes and Rist, 2008). When using polynomial basis functions of first or second order (bilinear, bi-quadratic) for the spatial data interpolation or regression such as bilinear or quadratic, only a monotonic velocity field or at most a distribution with a single peak inside the gap can be reconstructed.

The present work investigates the suitability of using the unsteady Navier-Stokes equations for incompressible flows to accurately estimate the velocity field in data gaps using physical modelling. The investigation is conducted making use of high-repetition rate PIV images obtained from experiments, where the objective is to reconstruct the time-varying velocity field inside the gaps. Firstly gaps are simulated inside the measurement domain in order to assess the validity of the method. Secondly the case of real large gaps caused by illumination shadows is considered.

\section{Working principle}

The case of two-dimensional incompressible flow will be considered. A flow is considered two-dimensional when one velocity component is negligible with respect to the other two. In planar PIV, the two velocity components $u$ and $v$ lying within the plane defined by the laser sheet are measured, while the out-of-plane component $w$ is not. The present technique employs a two-dimensional approximation of the flow field, assuming that the out-of-plane velocity component is negligible. To verify the validity of this hypothesis, the magnitude of $w$ can be estimated through the correlation peak height or the signal-to-noise ration SNR (ratio between highest and second highest peaks of the correlation map). When the out-of-plane velocity component is of the same order of the in-plane components, the hypothesis of two-dimensional flow does not hold and the application of a two-dimensional assumption would lead to inaccurate results.

In figure 1, a concrete example of experiment where the PIV data is not available in the entire measurement domain is shown: the velocity is measured in the domain $\mathscr{D}$, whereas the domain $\Omega$ is composed by the gaps $\Omega_{1}$ and $\Omega_{2}$ where no velocity information is present.

The measured velocity data are available in $\mathcal{D}$ at a given temporal rate $f_{\text {acq }}$ from the PIV experiment, whereas in $\Omega=\Omega_{1} \cup \Omega_{2}$ zero information is available on the spatio-temporal velocity distribution. The velocity in $\Omega$ is computed by solving the unsteady Navier-Stokes equations, which in non-dimensional form read:

$$
\begin{aligned}
& \nabla \cdot V=0 \\
& \frac{\partial \boldsymbol{V}}{\partial t}+\nabla p=\boldsymbol{R}
\end{aligned}
$$

where the vector $\boldsymbol{R}$ contains the contributions of advection and diffusion. 

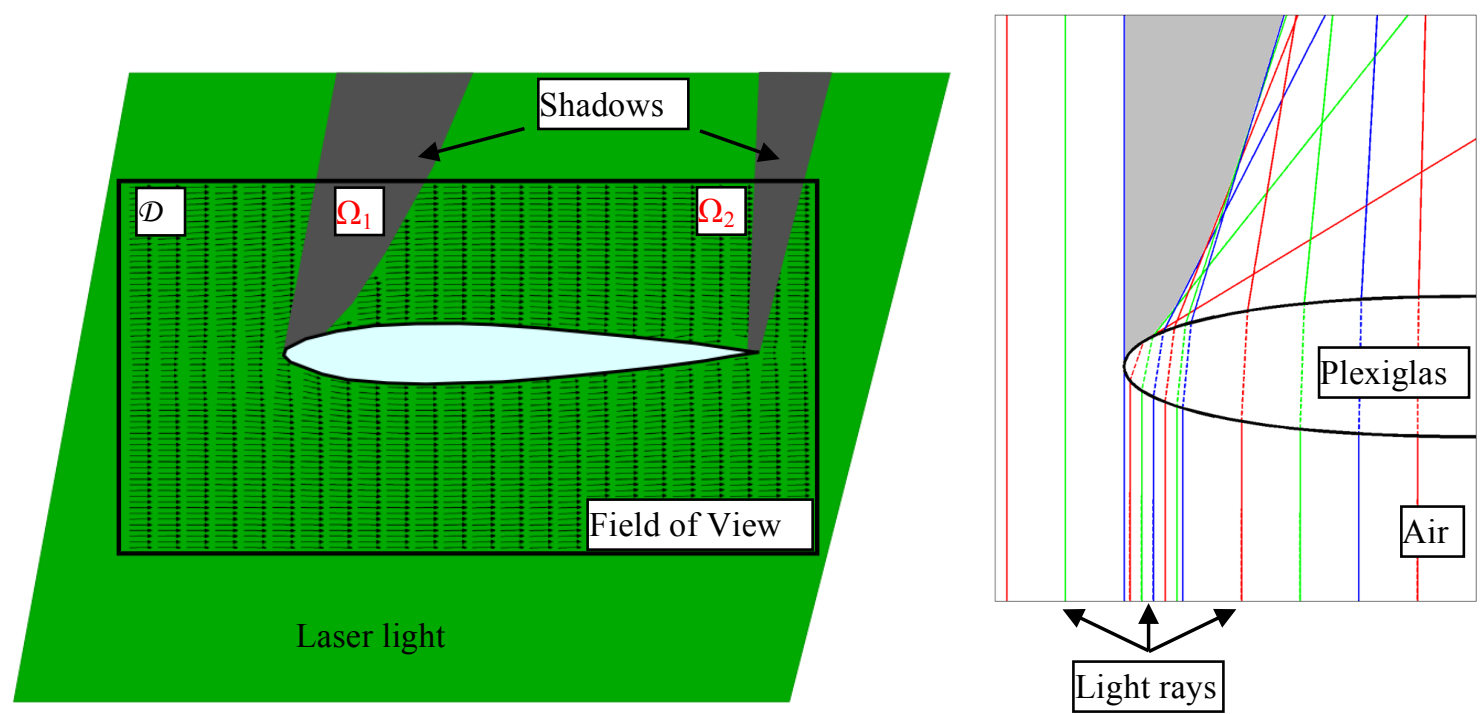

Figure 1. Left: schematic representation of measurement domain $(\mathscr{D})$ with two gaps $\left(\Omega_{1}\right.$ and $\left.\Omega_{2}\right)$; right: shadow region generated above the airfoil leading edge due to refraction.

The continuity equation (1) and the momentum equation (2) are combined to reformulate the problem as the Poisson equation for pressure (3):

$$
\Delta p=\boldsymbol{\nabla} \cdot\left(-\frac{\partial \boldsymbol{V}}{\partial t}+\boldsymbol{R}\right) .
$$

A staggered-grid finite volume method is employed to compute the pressure from (3); the velocity at the next time instant is obtained from a time-integration of (2). Time-varying Dirichlet boundary conditions on the velocity are imposed along $\partial \Omega$; in contrast, no boundary condition on the pressure is required. Furthermore, an initial condition on the velocity is required inside the gap, which is typically unknown. Therefore, the initial condition is chosen as a spatial interpolation of the velocity at the boundary by a bi-cubic function. If the flow is convection-dominated, the influence of the (erroneous) initial condition on the time-dependent solution vanishes after a time interval that scales with the ratio between the gap characteristic length and the advective velocity. After this time interval, the solution is predominantly determined by the boundary conditions as will be demonstrated.

\section{Treatment of boundary and initial conditions}

Equations (2) and (3) can be solved imposing boundary conditions only on the velocity and not on the pressure (Ferziger and Peric, 2002). The boundary conditions on $\boldsymbol{V}$ are provided at discrete time instants by the PIV measurements and are imposed as Dirichlet conditions. In order for the numerical scheme to be stable, the spacing $h$ of the computational mesh and the numerical time step $\delta t$ are selected according to the conditions proposed by Ferziger and Peric (2002) for the linear convection-diffusion equation. To fulfil those conditions, $\delta t$ often needs to be smaller than the time interval $\Delta t$ between PIV recordings. Therefore, the velocity boundary conditions at intermediate time instants are computed through interpolation in time of the PIV velocity data. The time interpolation herein employed is based on the advection model proposed by Scarano and Moore (2011). In general, the velocity boundary conditions are inexact because they contain random noise; as a consequence, an unphysical boundary layer or wiggles may be generated at the outflow boundaries. However, for Reynolds numbers $R e>>1$ the erroneous information at the outflow boundaries has only minor 
influence on the numerical solution, because it propagates upstream only over a distance $\mathrm{O}\left(\operatorname{Re}^{-1}\right)$ (Wesseling, 2001).

In contrast to the boundary conditions, the initial condition in the simulated domain is typically completely unknown. It will be shown numerically that after a time interval $\tau$, which scales with the ratio between the gap's characteristic dimension and the advection velocity, the solution in $\Omega$ is no longer sensitive to the initial condition and is determined predominantly by the boundary conditions. We therefore choose as initial condition a bicubic spatial interpolation of the boundary conditions at the initial time level. It is assumed that the measurement is performed over a time longer than $\tau$ such to reach independence from the initial condition.

\section{Algorithmic assessment}

To assess the technique, an artificial gap is constructed in existing full-field PIV data available from a study at the Aerodynamics Laboratories of TU Delft. Time-resolved planar and tomographic PIV measurements are performed on a circular jet in water. The jet exits a $10 \mathrm{~mm}$ diameter nozzle at $V_{r e f}=0.45 \mathrm{~m} / \mathrm{s}$ (the diameter-based Reynolds number is $R e_{D}=5,000$ ). A time-resolved sequence of snapshots is recorded in continuous mode at acquisition frequency of $1.2 \mathrm{kHz}$. A detailed description of the experiment is reported by Violato and Scarano, 2011. A region of $440 \times 440$ pixels resolution centered along the jet axis and extending between $D$ and $3 D$ from the nozzle exit is considered; $17 \times 17$ pixel interrogation windows with a vector pitch $h$ of 4 pixels $\left(h=h_{x}=h_{y}=0.25 \mathrm{~mm}\right)$.

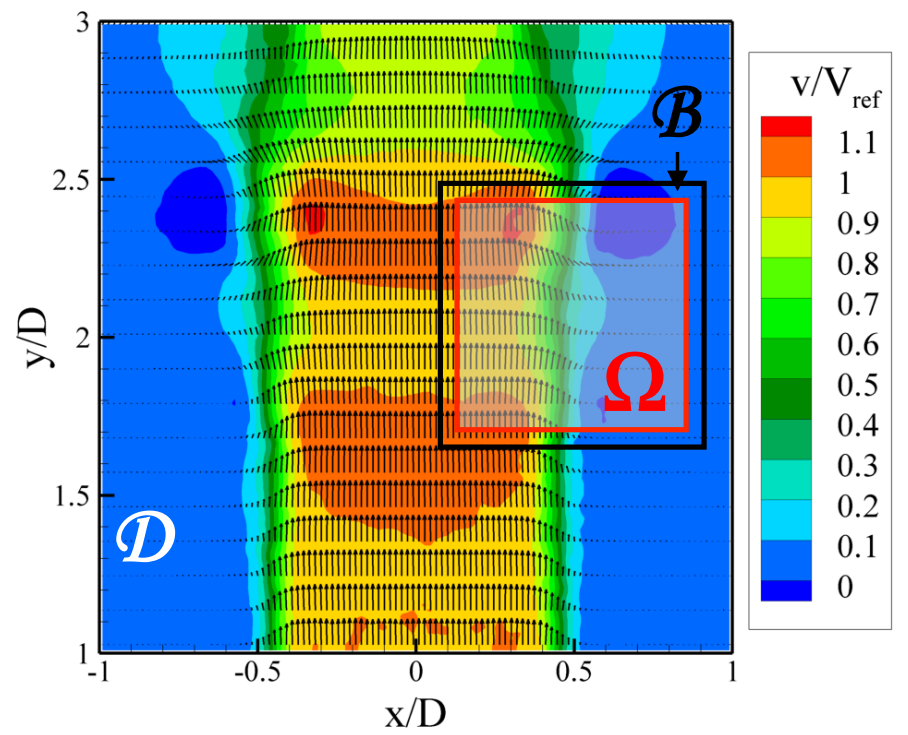

Figure 2. Laminar flow issued from a circular jet; axial velocity $v$. D: measurement domain; $\Omega$ : simulated gap; $\mathbb{B}$ : buffer region.

A data gap is simulated in the velocity field across the shear layer. The simulation is performed at the same Reynolds number as the flow $\left(R e_{D}=5,000\right)$ in the domain $\Omega \cup \mathrm{B}$ (see figure 2) of size $L_{x} \times L_{y}=40 \times 40$ PIV grid nodes; this region corresponds to $1.3 \lambda \times 1.3 \lambda$, with $\lambda=0.55 D$ the wavelength of the large-scale vortices in the shear layer. In the following, the ratio between the numerical domain dimensions and $\lambda$ is indicated with $L^{*}$ and $L^{*}{ }_{y}$. The numerical mesh is composed of $400 \times 400$ cells. 
The region $\Omega$ simulates a gap where no data is available: here the numerical solution is computed without exploiting any information about the local experimental velocity. In contrast, $\mathscr{B}$ represents a buffer between PIV data and simulated data employed to compute the boundary conditions. Furthermore, in this region the velocity is obtained as a distance weighted linear combination of experimental and numerical data. After performing the numerical simulation, the computed velocity components and pressure are projected onto the original Cartesian grid of the PIV data.

To run the numerical simulation, the initial condition on the velocity $\boldsymbol{V}_{h}^{0}$ must be prescribed. However, since in $\Omega$ no velocity data is acquired, the initial condition is not provided by PIV measurements and has to be estimated, for instance as a uniform velocity or as an interpolation of data at the gap boundary. The prescribed initial condition affects only the solution $\boldsymbol{V}_{h}^{n}$ defined in the successive time interval $\tau$, i.e. for $n \delta t \leq \tau$; after $\tau, \boldsymbol{V}_{h}^{n}$ becomes predominantly independent of $\boldsymbol{V}_{h}^{0}$. Therefore, the choice of the initial condition is largely irrelevant for the solution after $\tau$. For a flow where a convective component is present in the domain, such time scale can be simply estimated as $\tau \approx \frac{L}{\boldsymbol{V}_{\text {ref }} \cdot \Phi}$, where $\boldsymbol{V}_{\text {ref }}$ is the advection velocity and $L$ is the dimension of the domain. The inner-product on the denominator indicates the projection of the advection velocity along the direction where the domain size is $L$. For flows with no dominant direction (e.g. recirculating regions within the gap), the value of $\tau$ is expected to be substantially larger (above one order of magnitude) and can be estimated as $\tau \approx L^{2} / v$, with $v$ the kinematic viscosity of the flow (Bird et al., 1976).

In this session, the time scale $\tau$ is evaluated experimentally by employing two different initial conditions, namely a uniform field of zero velocity and bicubic interpolation of the velocity at the gap boundary. This is done to evaluate first whether the advanced time solution has any dependence upon the initial conditions and also to explore the possibility of accelerating the initial condition independence of the solution. The temporal evolution of the velocity within the simulated data gap is measured and will be considered as reference for validating the simulation.

The spatio-temporal evolution of the velocity field computed from the uniform and interpolated initial conditions is shown in Figure 3; the time is expressed in the non-dimensional form $t=t_{\text {dim }} \times V_{\text {ref }} L$, being $V_{r e f}$ a reference advective velocity (in the present case it is chosen as the jet velocity at the nozzle exit, $\left.V_{\text {ref }}=0.45 \mathrm{~m} / \mathrm{s}\right), t_{\text {dim }}$ the dimensional time and $L$ a characteristic dimension of the numerical domain $(L=7.3 \mathrm{~mm})$. The flow is characterized by a sequence of vortices entering the simulated domain from the center of the lower boundary, advecting upwards with approximately constant speed, and exiting at the top boundary. 


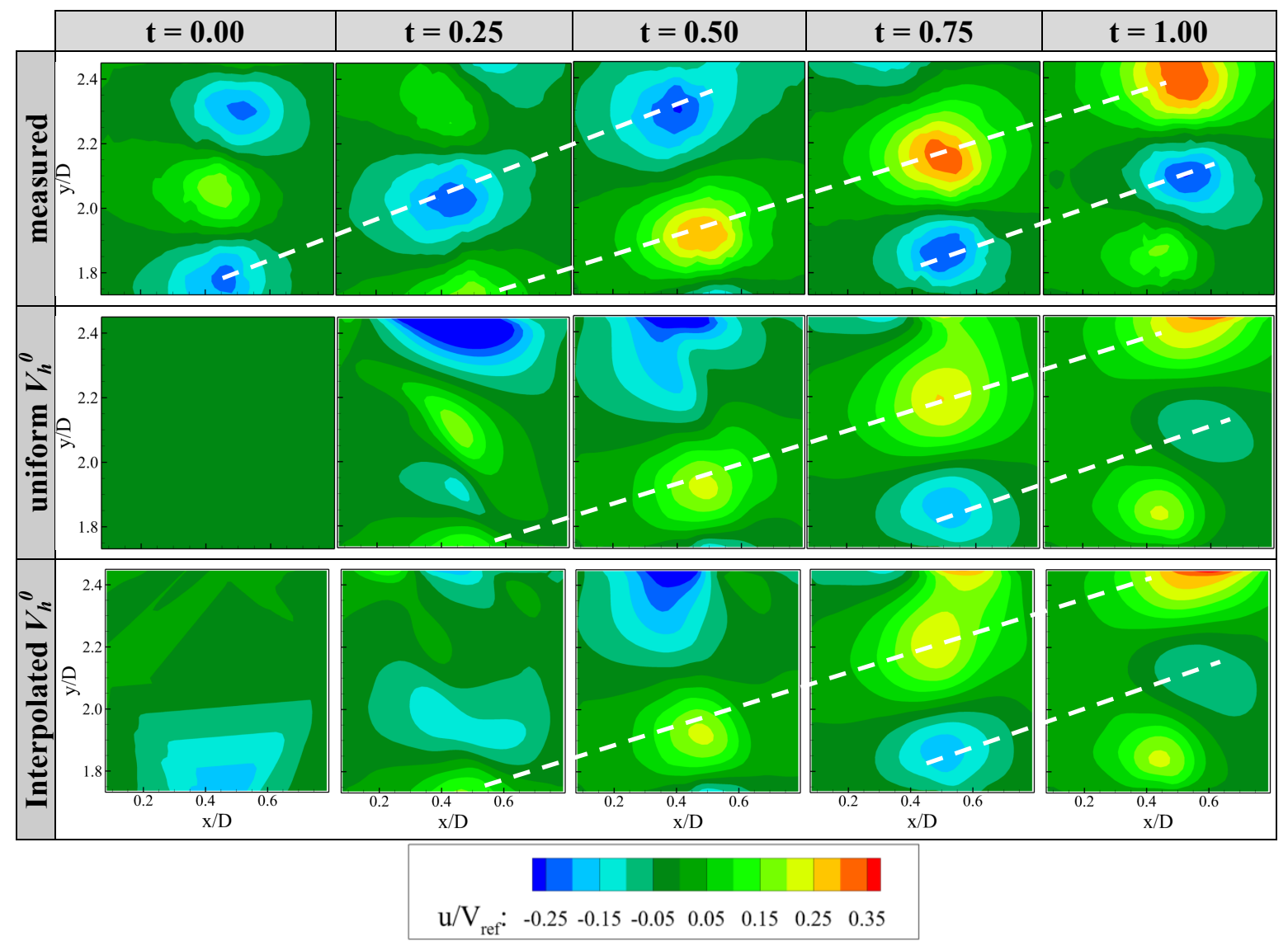

Figure 3. Contours of the computed radial velocity $u$ starting from different initial conditions (first row: measured velocity field; second row: uniform zero velocity initial condition; third row: initial condition as a bicubic interpolation of the boundary conditions). The white dashed lines indicate the temporal evolution of the flow structures.

Although the bicubic interpolation of the velocity is a closer approximation of the solution at $t=0$ than the uniform stagnant flow, it cannot be considered as an acceptable representation of the flow. After a non-dimensional time of 0.25 , the numerical result does not show a visible similarity with the experimental flow field. At $t=0.5$, about half the domain length has been swept by the flow information advected by the bottom boundary. As a consequence, the flow simulation resembles more the reference data in the lower half of the domain. In the upper half, the solution still depends on the initial conditions, therefore the numerical simulation is not able to reproduce the measured flow structure. The dependency on the initial conditions becomes less pronounced at $t \geq 0.75$, where the velocity contours exhibit only minor differences in shape. In particular, at $t=1$ the numerical simulations starting from different initial conditions produce nearly the same result.

The results obtained with the Navier-Stokes solver are presented in comparison with a bicubic interpolation of the gap boundary, which is more accurate than the common linear interpolation technique used in PIV to refill missing data (Westerweel ,1994, Nogueira et al., 1997, Raffel et al. 2007). In the numerical simulation, the initial condition is computed as a bicubic interpolation of the boundary conditions at $t=0$. In figure 4 , the reconstructed instantaneous velocity and vorticity fields are reported together with the experimental measurements. 

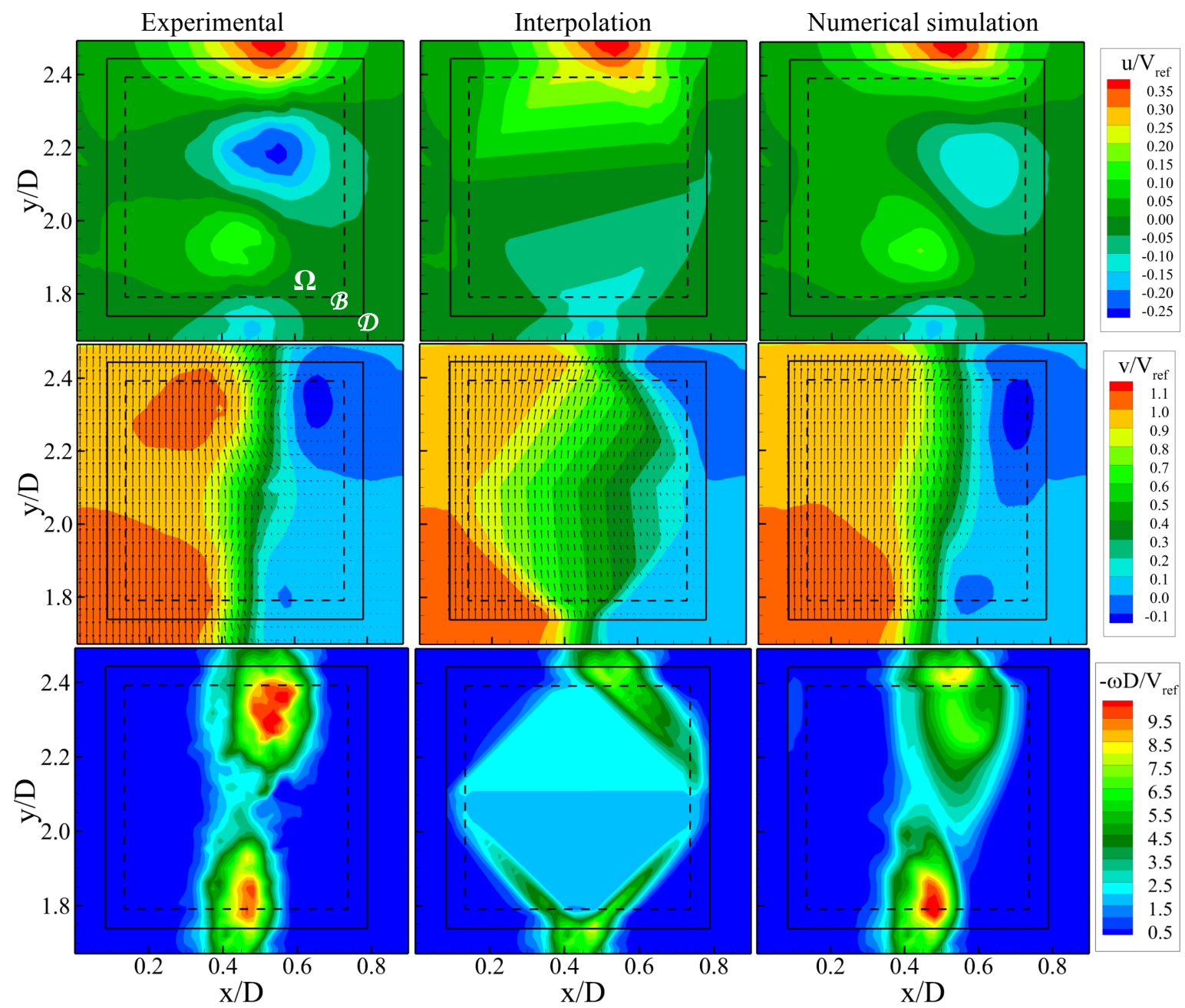

Figure 4. Instantaneous velocity field at $t=3.4$; first row: radial velocity; second row: axial velocity; third row: vorticity. From left to right: experimental result, bicubic interpolation of the boundary conditions, numerical simulation.

The velocity field selected for the experimental assessment presents a vortex of $0.5 \mathrm{D}$. The radial velocity measured by PIV shows a positive and a negative peak values. The bicubic interpolation is not able to reproduce the flow structure within the numerical domain, while the numerical simulation based on Navier-Stokes equations allows reconstructing it with the two peaks: the positive peak velocity does not show major differences from the experimental one, while damping effects up to $40 \%$ are noticed for the negative peak velocity. This discrepancy can be attributed to the fact that the negative peak velocity is further away from the inflow boundary, and numerical dissipation has reduced its strength. Similar conclusions can be drawn for the axial velocity contour, which is dramatically widened by the interpolation, and for the vorticity contour, where the numerical simulation reproduces the inflow peak within 7\% accuracy, while the outflow peak is significantly dumped by dissipation (reduction of 35\% with respect to the experimental value).

To quantify the error due to bicubic interpolation and numerical reconstruction, the root-mean-square (rms) error (Fincham and Delerce, 2000) with respect to the PIV velocity measurements in $\Omega$ is plotted as a function of the non-dimensional time $t$, see figure 5 left. 

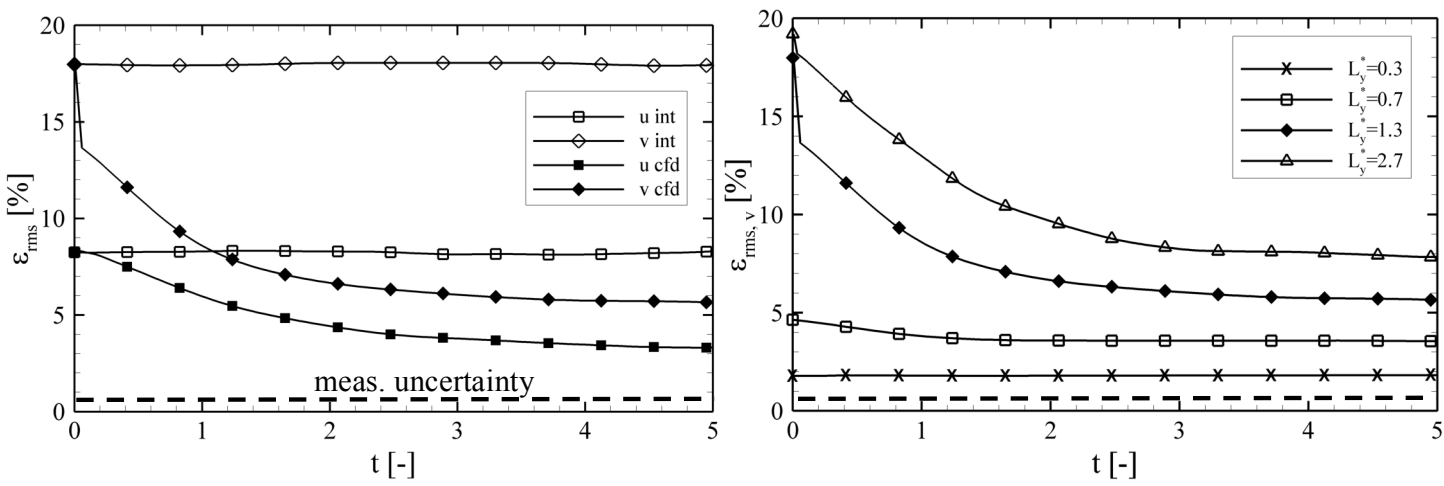

Figure 5. Root-mean-square error (expressed in percent of the reference velocity) as a function of $t$. Left: comparison between numerical simulation (cfd) and bicubic interpolation (int); right: test for different domain sizes. Dashed line: measurement uncertainty.

When the velocity field in $\Omega$ is computed through a bicubic interpolation of the boundary conditions, the root-mean-square error is approximately constant in time and equals $8.2 \%$ and $18.0 \%$ of $V_{r e f}$ for the radial and axial velocity components respectively. On the contrary, the error of the numerical simulation decreases in time reaching a plateau after $t=3$, where $\varepsilon_{\mathrm{rms}}$ for the radial and axial velocity components equals $3.3 \%$ and $5.7 \%$ of $V_{\text {ref }}$ respectively; this reduction is due to the diminishing influence of the (erroneous) initial condition on the numerical result.

The effect of the numerical domain size on the accuracy of the results is investigated in figure 8 right. The rms error for only the axial velocity component is reported; analogous results are obtained for the radial velocity component. The height of the domain is set to 10, 20, 40 and 80 PIV grid points respectively $\left(L^{*}{ }_{y}=0.3,0.7,1.3\right.$ and 2.7 respectively), while the width is kept constant at 40 PIV grid points $\left(L^{*}=1.3\right)$. The plot of figure 8 right shows that the accuracy of the simulation is strongly related to the size of the numerical domain. When the domain's height is $L_{y}{ }^{*}=0.3$, the discrepancy between numerical and experimental data is $1.8 \%$; this value increases up to $7.8 \%$ for $L_{y}{ }^{*}=2.7$.

\section{Application: treatment of shadow regions}

The technique is applied to an experiment in air of a rod-airfoil configuration. A cylindrical rod of $6 \mathrm{~mm}$ diameter is mounted $10.2 \mathrm{~cm}$ upstream of a NACA 0012 airfoil, having a chord of $10 \mathrm{~cm}$ and placed at zero angle of attack. The airfoil is made out of Plexiglas in order to be transparent to the laser light. The nominal free-stream velocity is set to $15 \mathrm{~m} / \mathrm{s}$, yielding Reynolds numbers of 6,000 and 100,000 with respect to the rod diameter and the airfoil chord respectively. The temporal separation between laser pulses is $50 \mu \mathrm{s}$, while the acquisition frequency is $2,700 \mathrm{~Hz}$. The field of view size is $164 \times 83 \mathrm{~mm}$, imaged by $1939 \times 1024$ pixels. A thorough description of the experiment is reported in Lorenzoni et al., 2009.

The illumination is directed from the bottom of the field of view upwards, tilted clockwise by 11 degrees. Two shadow regions are produced above the leading and trailing edges due to refraction effects; in these regions, indicated with $\mathrm{A}$ and $\mathrm{B}$ respectively in figure, no particle images are visible and no displacement vector can be extracted through a correlation-based PIV algorithm. 

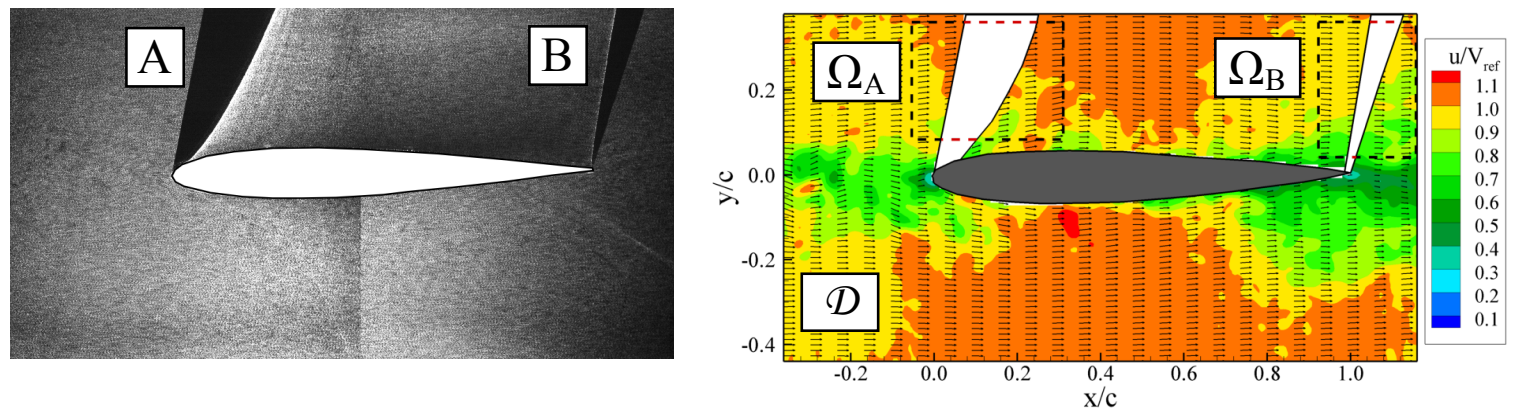

Figure 6. Left: double-frame recording of particle images on a transparent NACA 0012 airfoil (laser light inserted from the bottom) in the wake of a rod (outside the field of view). Right: numerical domain $\Omega$ and the regions A and B. The PIV velocity vectors are plotted every 10 sample in the horizontal direction and every 2 samples in the vertical direction.

The images are analysed with $32 \times 32$ pixels interrogation windows with $75 \%$ overlap, yielding a vector pitch of 8 pixels. For both the shadow regions $\mathrm{A}$ and $\mathrm{B}$, the numerical simulation is conducted in rectangular domains, indicated by $\Omega_{\mathrm{A}}$ and $\Omega_{\mathrm{B}}$ respectively. $\Omega_{\mathrm{A}}$ is composed by $112 \times 88$ cells, corresponding to $56 \times 44$ PIV grid nodes, while $\Omega_{\mathrm{B}}$ is composed by $62 \times 100$ cells, which correspond to $31 \times 50$ PIV grid nodes. The time step is set to $1 / 40^{\text {th }}$ of the temporal separation between image pairs. The simulation is run at Reynolds number 1,000 (based on the airfoil chord) to add numerical dissipation in order to stabilize the solution at the chosen time step and mesh size.

Both numerical domains $\Omega_{\mathrm{A}}$ and $\Omega_{\mathrm{B}}$ exhibit a central region where no PIV data is present and two lateral regions where particle image velocimetry vectors have been computed; the numerical solution is calculated in the entirety of the rectangular domains, but it is retained only in the central regions. At the unknown boundaries (the red dashed lines of figure 6), the boundary conditions are computed as a linear interpolation of the measured PIV velocity data.

To investigate the accuracy of the method, the statistical results (mean and fluctuations root-mean-square) obtained from the reconstructed velocity fields are shown in figure 7 . The mean horizontal and vertical velocity components (figure $7 \mathrm{a}$ and $\mathrm{b}$ respectively) calculated in the shadow regions preserve the symmetry of the flow field with respect to the airfoil. The uncertainty in the mean components is estimated to be below $0.6 \%$ of $V_{\text {ref }}$ (i.e. 0.05 pixels). In contrast, the fluctuations root-mean-square contours exhibit slight discontinuities between numerical and experimental values; the uncertainty on those components is of the order of $1 \%$ of $V_{\text {ref }}$ (i.e. 0.09 pixels).

The added value of the present technique with respect to interpolation approaches is evident when small flow structures need to be reconstructed in the shadow region. In figure 8 the motion of two vortices $a$ and $b$ is illustrated. At time $t=0$, vortex $a$ has peak vorticity (normalized with respect to $V_{\text {ref }}(c)$ of magnitude 13.4; in contrast, vortex $b$ has two distinct cores $0.037 c$ apart with peak vorticity of 18.6 and 10.3 respectively. The two vortices are advected downstream by the flow and pass through the shadow region, where the velocity is calculated via the numerical simulation.

The proposed filling approach allows tracking the vortices within the shadow region. However, modulation effects are noticed which have two main consequences: reducing the peak vorticity up to $50 \%$ of the original value and merging the two cores of vortex $b$ in a single-core vortex. Downstream of the shadow region, the vorticity is measured from PIV data: the peak vorticity is recovered and the two cores of vortex $b$ become distinct again. Minor modulation effects also occur in the immediate vicinity of the shadow region edges, where the velocity is computed as a distance-weighted combination of experimental and numerical values. 

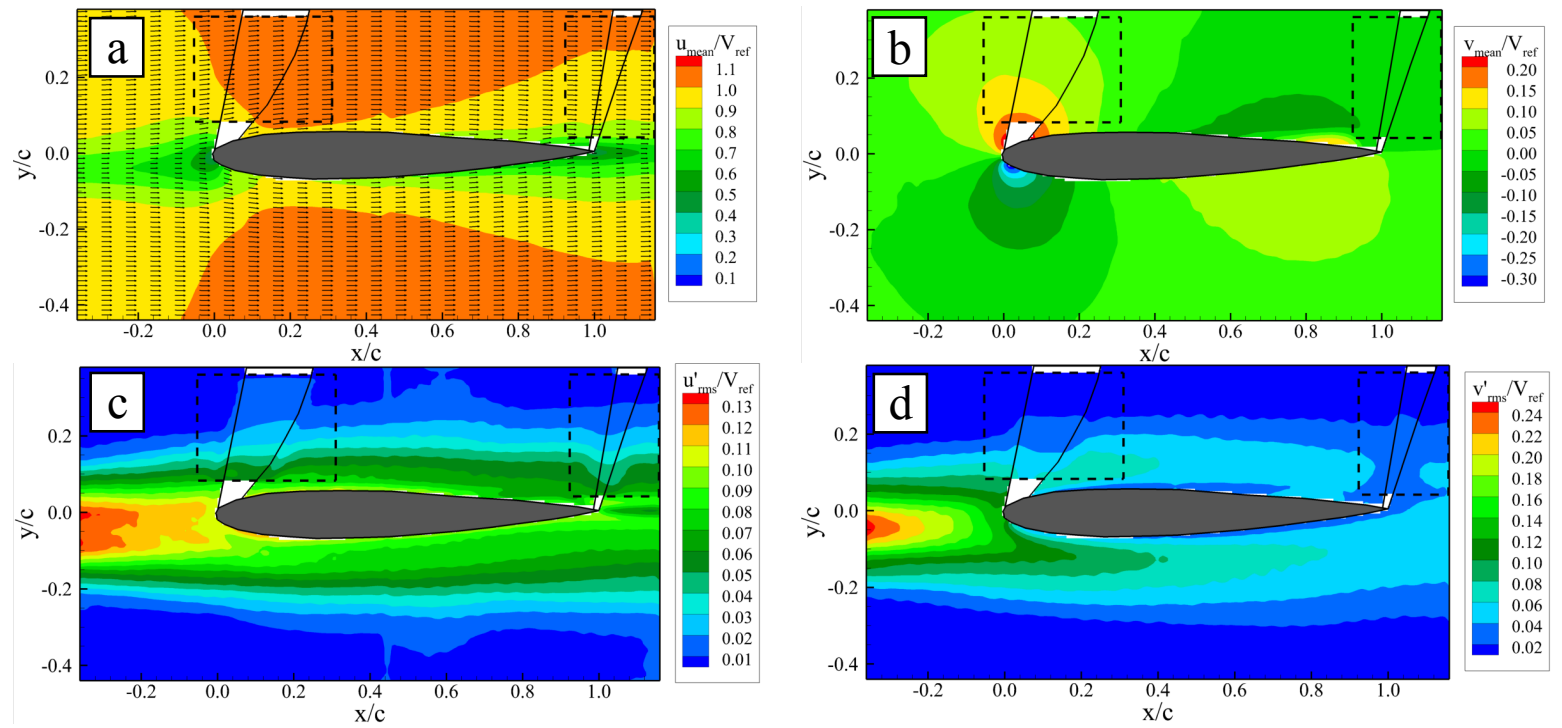

Figure 7. Statistical results: a) mean horizontal velocity; b) mean vertical velocity; c) velocity fluctuations in the horizontal direction; d) velocity fluctuations in the vertical direction.

In contrast, when the velocity in the shadow region is computed via bicubic interpolation of the boundary values, the motion of the two vortices along the trajectory cannot be tracked, as illustrated in figure 8 right.

\section{Conclusions}

A novel technique for filling in gaps in time-resolved PIV data has been proposed. The technique takes as input the measured velocity values at the gap boundary and solves the unsteady incompressible Navier-Stokes equations within the gap. The finite volume approach is employed with central discretization of the convective and diffusive terms and explicit forward discretization in time. Since the required numerical time step is usually smaller than the time interval between the measured PIV velocity fields, the boundary conditions are interpolated in time employing an advection-based model. The assessment of the technique on an artificial gap constructed in existing full-field PIV data has shown that the accuracy of the results depends on the numerical domain size. In typical conditions of applicability (PIV data sufficiently resolved in time and numerical domain size of the order of the wavelength of the large-scale vortices in the flow), the accuracy of the method is within 5\% of the free-stream velocity. By using PIV data with real gaps, the capability of the method of reconstructing the velocity field where no velocity information is available has been shown.

\section{Acknowledgements}

The work is carried out in the framework of the Adaptive PIV project funded by LaVision GmbH. 

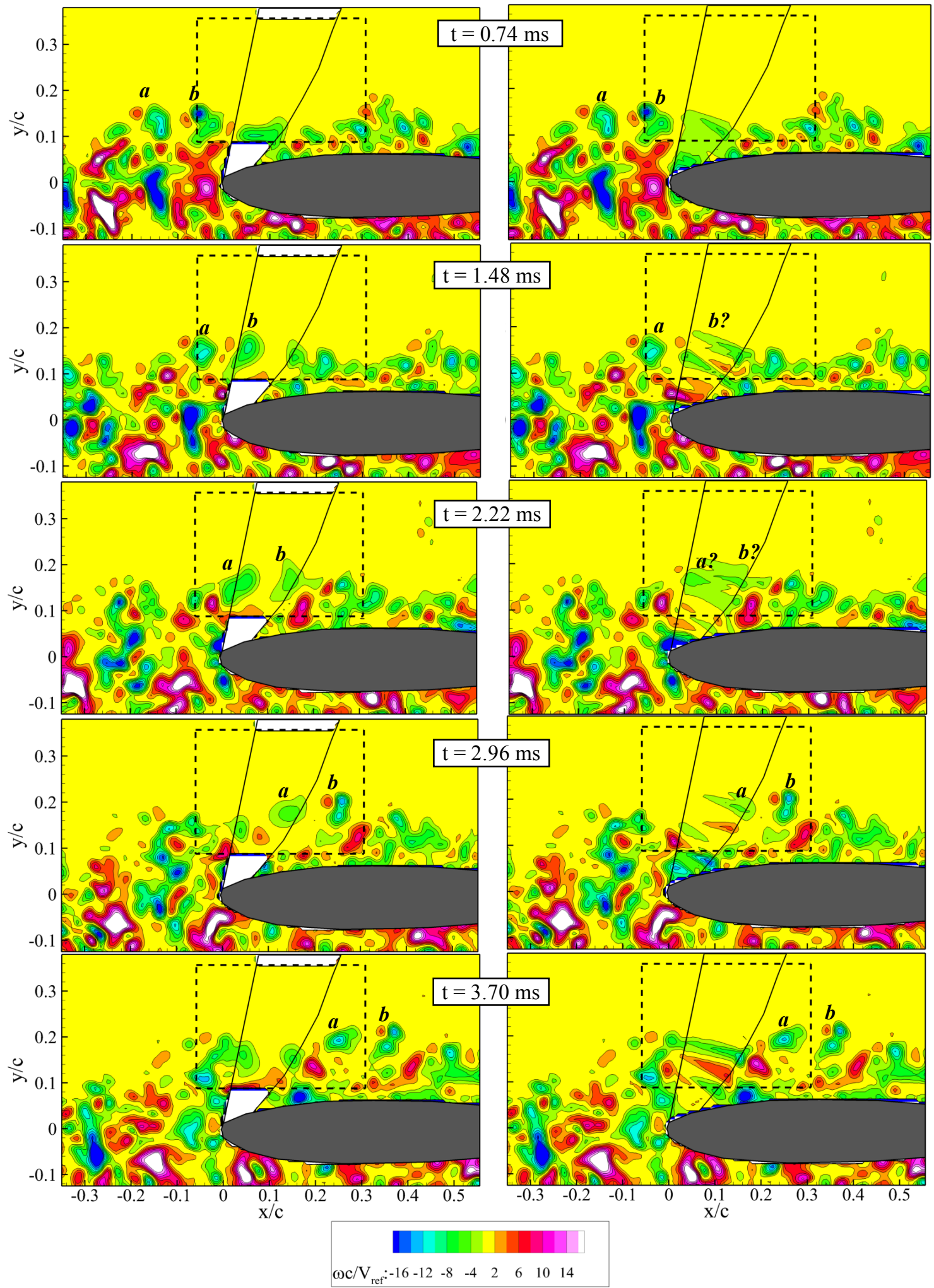

Figure 8. Vorticity contours showing the advection of two vortices $a$ and $b$ through the shadow region. Left: reconstruction through the Navier-Stokes solver; right: reconstruction through a bicubic interpolation of the velocity boundary values. 


\section{References}

Bird RB, Stewart WE, Lightfoot EN (1976), Transport phenomena, John Wiley \& sons.

Ferziger JH, Peric M (2002), Computational methods for fluid dynamics, Springer.

Fincham A, Delerce G (2000), Advanced optimization of correlation image velocimetry algorithms, Exp. Fluids (Suppl.), S13-S22.

Garcia D (2011), A fast all-in-one method for automated post-processing of PIV data, Exp. Fluids 50:1247-1259.

Gunes H, Rist U (2008), On the use of kriging for enhanced data reconstruction in a separated transitional flat-plate boundary layer, Physics of Fluids 20, 104109.

Kurtulus DF, Scarano F, David L (2007), Unsteady aerodynamic forces estimation on a square cylinder by TR-PIV, Exp. Fluids 42:175-196.

Lorenzoni V, Tuinstra M, Moore PD, Scarano F (2009), Aeroacoustic analysis of a rod-airfoil flow by means of time-resolved PIV, $15^{\text {th }}$ AIAA/CEAS Aeroacoustic Conference $\left(30^{\text {th }}\right.$ AIAA Aeroacoustic Conference), Miami, Florida, May 11-13, 2009.

Nogueira J, Leucona A and Rodriguez PA (1997), Data validation, false vectors correction and derived magnitudes calculation on PIV data, Meas. Sci. Technol. 8, 1493-1501.

Raffel M, Willert CJ, Wereley ST, Kompenhans J (2007), Particle image velocimetry - a practical guide, Springer.

Scarano F and Moore PD (2011), An advection-based model to increase the temporal resolution of PIV time series, Exp Fluids, DOI 10.1007/s00348-011-1158-3.

Scarano F, Sciacchitano A (2011), Robust elimination of light reflections in PIV, $9^{\text {th }}$ International Symposium on Particle Image Velocimetry PIV'11, Kobe, Japan, July 21-23, 2011.

Theunissen R, Scarano F, Riethmuller ML (2009), On improvement of PIV image interrogation near stationary interfaces, Exp. Fluids 45:557-572.

Violato D, Scarano F (2011), Three-dimensional evolution of flow structures in transitional circular and chevron jets, Physics of Fluids 23,124104.

Wesseling P (2001), Principles of computational fluid dynamics, Springer.

Westerweel J (1994), Efficient detection of spurious vectors in particle image velocimetry data, Exp. Fluids 16:236-247

Westerweel J, Scarano F (2005), Universal outlier detection for PIV data, Exp. Fluids 39:1096-1100. 